\title{
Módulo Terminal Remoto, para la adquisición de datos, monitoreo y control de procesos Agroindustriales - AgriculTIC
}

\author{
Remote Terminal Module, for data acquisition, monitoring and \\ control of Agro-industrial processes - AgriculTIC. \\ Jorge Enrique Chaparro Mesa ${ }^{1 *} \quad$ Nelson Barrera Lombana ${ }^{2} \quad$ Fredy Alonso León Socha ${ }^{3}$ \\ Recibido 3 de abril de 2019, aceptado 9 de junio de 2020 \\ Received: April 3, 2019 Accepted: June 9, 2020
}

\begin{abstract}
RESUMEN
Este artículo describe el desarrollo de un Módulo Terminal Remoto, escalable y compatible con diversidad de sensores y actuadores disponibles en el mercado. El dispositivo fue diseñado para automatización y monitoreo de procesos agroindustriales, con posibilidad de intervención en sitio o a través de servicios de Internet de las cosas (IoT). El sistema cuenta con puertos digitales y analógicos programables, USB On-The-Go (OTG) y bus 1-wire para conexión de diferentes sensores (temperatura, $\mathrm{pH}$, humedad relativa y nivel de oxígeno, entre otros); en cuanto a los actuadores se configuran en dependencia de eventos, o calendario electrónico por reloj de tiempo real (RTC). El registro de datos se realiza de forma local o mediante servicios de computación en la nube, a través de un sistema de comunicación compatible con protocolos TCP/IP, GSM, SMS, EGPRS, 3G y 4G. Por su parte el acceso al sistema se establece de forma remota, o local, a través de dispositivos USB con funcionalidad OTG o Bluetooth. El módulo está compuesto por tres subsistemas independientes; software, hardware y módulo de comunicación. El ciclo de vida del Proyecto se llevó a cabo mediante un modelo en V; integrando la metodología SCRUM para el desarrollo de la plataforma software. Así mismo el módulo de comunicación se desarrolló mediante un protocolo abierto, que permite a otros desarrolladores, generar nuevas soluciones tecnológicas e incorporar otras funcionalidades al sistema. La validación del sistema se realizó bajo el estándar IEEE 1012-2016 y las pruebas de funcionamiento del prototipo se efectuaron en cultivos agrícolas de la región de Casanare-Colombia.
\end{abstract}

Palabras clave: Variables agrometeorológicas, monitoreo en tiempo real, Internet de las cosas, agricultura de precisión, sensores, adquisición de datos, Bus 1-wire.

\section{ABSTRACT}

This article describes the development of a Remote Terminal Module, scalable and compatible with the diversity of sensors and actuators available in the market. The device was designed for automation and monitoring of agro-industrial processes with the possibility of intervention through the Internet of Things (IoT) services or commands on the site. The system has programmable digital and analog ports, USBOTG, 1 -wire bus for connection of different sensors, and configurable actuators depending on events or

1 Grupo de investigación SISTEMIC. Universidad de Antioquia. Grupo de investigación TICTROPICO, Unitrópico. Carrera 19 No39-40 Yopal, Colombia. E-mail: jorgechaparro@unitropico.edu.co

2 Escuela de Ingeniería Electrónica. Universidad Pedagógica y Tecnológica de Colombia. UPTC. Calle 4 A Sur Nº 15-134. Sogamoso, Colombia. E-mail: nelson.barrera@uptc.edu.co

3 Dirección de proyectos. Centro de Desarrollo Tecnológico e Innovación en TIC. NetworkTIC. Calle 33 Nº 28-47. Yopal, Colombia. E-mail: gerencia@networktic.com

* Autor de correspondencia: jorgechaparro@unitropico.edu.co 
electronic calendar by real-time clock (RTC). The data is recorded locally or through cloud computing services through a communication system that transmits through TCP/IP, GSM, SMS, EGPRS, 3G, and $4 G$ protocols. The system's connection is made remotely or locally using USB with functionality OTG or Bluetooth devices. The device was built in a modular way and comprised of three subsystems; software, hardware, and communication module. The software was developed with a Scrum Methodology integrated with a V model for the system's general development. The communication module was developed with an open protocol that allows other developers to generate new technological solutions and incorporate other functionalities. The validation was carried out according to the parameters established by the IEEE 1012-2016 standard and, the operation tests of the prototype were carried out on agricultural crops in the region of Casanare-Colombia.

Keywords: Agrometeorological variables, monitoring in real-time, Internet of things, precision agriculture, sensing, data acquisition, 1-Wire bus.

\section{INTRODUCCIÓN}

La implementación de nuevas tecnologías al sector agropecuario, ha sido fundamental para mitigar los problemas relacionados al cambio climático; el cual ejerce presión sobre la genética de las plantas y minimiza la capacidad productiva de las tierras [1]. Frente a esta situación surge como reto primordial, lograr innovaciones que permitan aumentar la productividad agrícola de forma sostenible, beneficiando a los productores y a su vez preservando los recursos naturales. En este sentido las Tecnologías de Información y Comunicaciones TIC y la electrónica, han aportado herramientas innovadoras al sector agropecuario, que han sido fundamentales en la producción y el manejo diferenciado de áreas agrícolas [2,3]. Este tipo de tecnologías se basan en "hacer lo correcto en el lugar adecuado y en el momento oportuno" $[4,5]$.

El desarrollo óptimo del sector agropecuario está relacionado directamente con el manejo adecuado de variables esenciales para el establecimiento del cultivo. Para este caso en particular, temperatura, humedad del suelo, aire y pH [6]. Sin embargo, mantener estas variables vigiladas y controladas constantemente, es un trabajo costoso y dispendioso, que requiere la presencia continua de un operario, ocasionando gastos de logística, infraestructura física e incrementos salariales entre otros. No obstante el no hacerlo implica riesgos en los cultivos, que ocasionan bajos rendimientos, mala calidad en los productos, pérdida de la trazabilidad de la información y pérdidas económicas [7].

En la actualidad existen diversas tecnologías que resuelven algunos de estos problemas; en su gran mayoría desarrolladas por empresas extranjeras, ocasionando gastos de importación, que, sumado a la carencia de soporte técnico, dificultan la adquisición y disminuyen el interés por parte de los productores; logrando que muchos de ellos opten por descartar estas opciones [8].

En este contexto surge esta iniciativa, como respuesta a la necesidad que tienen los agricultores de automatizar sus procesos agrícolas con tecnologías asequibles, adaptadas a las condiciones de los cultivos y respaldadas de un soporte técnico local.

Teniendo en cuenta esta problemática, se desarrolló el Módulo Terminal Remoto (MTR) para el monitoreo y la automatización de procesos agroindustriales, con posibilidad de intervención, mediante servicios de (IoT) $[9,10]$; o a través de una terminal de comandos en el sitio de la intervención. Este desarrollo tecnológico está conformado por puertos digitales y analógicos programables de uso general, bus 1-wire para conexión de transductores de temperatura, $\mathrm{pH}$, nivel de oxígeno y humedad relativa; además incluye actuadores configurables en dependencia de eventos programables, así mismo cuenta con sistema de almacenamiento local o mediante servicios de computación en la nube, ya que transmite los valores de las variables a través de la web o mediante tecnologías EGPRS. Por otra parte, el módulo permite realizar la configuración de parámetros en línea, o en el sitio, a través de dispositivos compatibles con puerto USB OTG o Bluetooth. En cuanto al sistema de seguimiento y control se pueden configurar las acciones a ejecutar de acuerdo con la cuantificación que se haga a 
las variables presentes en el sistema o mediante calendario electrónico por reloj de tiempo real (RTC).

La validación del prototipo se realizó en cultivos de piña y hongo de orellana, en la ciudad de Yopal, departamento del Casanare Colombia.

\section{ANTECEDENTES}

Con el ánimo de fundamentar el trabajo realizado, es indispensable contextualizar la propuesta investigativa, especialmente con trabajos conmensurables realizados en el hemisferio occidental y especialmente en las circunstancias particulares que se dan en Latinoamérica; en este sentido, se debe mencionar como relevante, que en Chile, se desarrolló un sistema web de bajo costo que permite realizar el monitoreo de un invernadero en forma constante vía web [11]. De igual forma en CORHUILA, Colombia, se desarrolló un sistema de control y automatización de temperatura, humedad del suelo y humedad relativa para optimizar el rendimiento de cultivos bajo cubierta [12]. Por su parte el centro agro empresarial y acuícola, del SENA, regional Guajira Colombia, se implementó un prototipo para optimizar la automatización de un sistemas de riego, mediante la utilización de estrategias de control a través de dispositivos móviles [13]. En la ciudad de Bogotá se desarrolló un dispositivo para monitorear en tiempo real vía web variables como temperatura, humedad y concentración de $\mathrm{CO} 2$, las cuales puedan afectar la calidad de la mercancía durante el transporte [14]. En este mismo sentido en Cuba se ha venido trabajando en sistemas de control y monitoreo en tiempo real, que permiten la operación remota de máquinas de riego de pivote central [15].

De igual forma, en Ecuador se desarrolló una red inalámbrica de sensores inteligentes que se encargan de supervisar, recolectar y transmitir información medida por sensores en tiempo real, permitiendo obtener datos de $\mathrm{pH}$, temperatura, conductividad y turbidez del agua, así como también humedad relativa y temperatura ambiente, del lugar en donde se realiza la supervisión [16]. Igualmente, en este país, en la ciudad de Ibarra se desarrolló una red inalámbrica de sensores con el fin de mejorar el rendimiento de los cultivos, esta red trabaja con el estándar 6loWPAN, y permite monitorear variables aplicadas en agricultura de precisión como: humedad relativa, temperatura, humedad de suelo, luz y lluvia [16]. En México en la Universidad Autónoma de Querétaro, desarrollaron un sistema de automatización basado en microcontroladores

Tabla 1. Módulos del sistema propuesto por Mamani, Villalobos and Herrera [11].

\begin{tabular}{|l|l|}
\hline \multicolumn{1}{|c|}{ Módulos } & \multicolumn{1}{c|}{ Descripción } \\
\hline $\begin{array}{l}\text { Recolección y } \\
\text { envío de datos }\end{array}$ & $\begin{array}{l}\text { Involucra un microcontrolador (placa NodeMCU) con acceso a internet y algunos sensores, } \\
\text { (temperatura AM2301, Iluminación BH1750, gas MQ135 y temperatura Sht10. Este } \\
\text { microcontrolador permite la extracción y manipulación de los datos desde los sensores, para } \\
\text { luego enviarlos al servidor de la aplicación. }\end{array}$ \\
\hline Servidor & $\begin{array}{l}\text { La aplicación en sí, que está a cargo de escuchar y recibir los datos enviados por el microcontrolador } \\
\text { de recolección, e insertarlas en la base de datos MySql. Además, es la encargada de enviar la } \\
\text { información solicitada a la vista del usuario o administrador cuando este la requiera. }\end{array}$ \\
\hline $\begin{array}{l}\text { Control de } \\
\text { dispositivos }\end{array}$ & $\begin{array}{l}\text { Involucra un microcontrolador, que controla los dispositivos ubicados en el invernadero como } \\
\text { el foco de leds, el que se prende como una señal de alarma cuando el módulo de recolección y } \\
\text { envío de datos detecta que los niveles de las variables a las que se les hace seguimiento están } \\
\text { sobrepasando los niveles máximos y mínimos permitidos, y la bomba de agua que es activada } \\
\text { por el usuario desde la web para regar los cultivos dentro del invernadero. }\end{array}$ \\
\hline $\begin{array}{l}\text { Seguimiento y } \\
\text { Control }\end{array}$ & $\begin{array}{l}\text { Vistas que muestran el histórico de los datos del medio ambiente recolectados hasta la fecha } \\
\text { y la opción de configurar ciertos parámetros. Por otro lado, muestran los datos obtenidos } \\
\text { por los sensores en tiempo real y permite controlar los dispositivos que están a cargo del } \\
\text { microcontrolador de control. }\end{array}$ \\
\hline CloudMQTT & $\begin{array}{l}\text { Es el servidor de mensajería que actúa como el canal de comunicación entre el usuario y los } \\
\text { microcontroladores a cargo de la recolección de datos y el control de dispositivos. CloudMQTT } \\
\text { actúa como un bróker entre estos dispositivos, permitiendo una interacción en tiempo real. }\end{array}$ \\
\hline
\end{tabular}

Fuente: Elaboración con base a [11]. 
de alto desempeño y tecnología ZigBee para el monitoreo y control de invernaderos de forma local y remota a través de internet [17].

Adicionalmente, de forma complementaria, se debe mencionar que en Asia, específicamente en Beijing China, se desarrolló un sistema inalámbrico remoto para el monitoreo en línea de la calidad del agua en un cultivo intensivo de peces [18]. La empresa King Pigeon en China desarrollo un dispositivo para el monitoreo y control de variables como temperatura y humedad, utiliza un protocolo Modbus GSM 3G 4G y a través de mensajes de texto envía alertas e información de las del estado de las variables [19]. Existen otros desarrollos similares para la automatización de sistemas de riego, los cuales monitorean y controlan remotamente desde un teléfono celular y utilizan tecnologías Arduino y Android, [20-24].

Para complementar, a continuación, se referencian algunos trabajos de forma puntual, que fueron base importante para la elaboración de la propuesta expuesta en el presente texto, esta revisión, es importante en la definición de derroteros que orientaron la construcción del hardware, del firmware y del software de AgriculTIC.

\section{Trabajos relacionados}

Trabajo 1. Sistema web de bajo costo que permite realizar seguimiento en línea de variables vitales para un cultivo en invernadero, permitiéndole al agricultor detectar oportunamente, condiciones adversas, que pueden afectar la producción para así determinar acciones, desde cualquier lugar donde se encuentre, ahorrando gastos en movilización y tiempo [11].

Trabajo 2. Dispositivo para monitorear en tiempo real variables que puedan afectar la calidad en el proceso de transporte, de mercancías [14]. Las generalidades se muestran en la Tabla 2.

Trabajo 3. Red inalámbrica de sensores que trabaja con el estándar 6loWPAN, permitiendo monitorear variables como, humedad relativa, temperatura, humedad de suelo, luz y lluvia.

\section{METODOLOGÍA DE DESARROLLO}

Las metodologías de desarrollo de sistemas embebidos, toman cada día más fuerza, ya que además de guiar el perfeccionamiento del software y el hardware con calidad, buscan la integración de protocolos y sistemas eficientes de comunicación, que permiten conectar dispositivos electrónicos a internet [25]. En el caso particular expuesto en este documento, se asume como sistema embebido a una aplicación de propósito específico, alojada en un procesador o en un dispositivo programable, que gestiona datos generados por sensores, realiza acciones de control sobre actuadores o transmite información en forma remota para realizar un seguimiento permanente [26].

Teniendo en cuenta que al revisar el estado del arte, no se encontró una metodología ampliamente aceptada para el desarrollo de sistemas embebidos $[27,28]$, se utilizaron diferentes metodologías del

Tabla 2. Módulos del sistema propuesto por Puentes Rivero y Baquero Cardozo [14].

\begin{tabular}{|l|l|}
\hline \multicolumn{1}{|c|}{ Módulos } & \multicolumn{1}{c|}{ Descripción } \\
\hline $\begin{array}{l}\text { Recolección y } \\
\text { envío de datos }\end{array}$ & $\begin{array}{l}\text { Se implementó con un computador de placa reducida (Raspberry Pi 3 modelo B) y un } \\
\text { microcontrolador (PIC18F4550) para realizar la adquisición de las señales, la interpretación } \\
\text { de datos y transmisión de información a internet. }\end{array}$ \\
\hline Servidor & $\begin{array}{l}\text { Servidor con soporte para PHP y MySQL por medio de Apache HTTP SERVER, con el fin de } \\
\text { obtener una página WEB con contenido dinámico. }\end{array}$ \\
\hline $\begin{array}{l}\text { Control de } \\
\text { dispositivos }\end{array}$ & $\begin{array}{l}\text { Este módulo, contiene un microcomputador, que tiene como tarea, el procesamiento de los } \\
\text { datos del sistema, cuantificando variables, determinando acciones y almacenando eventos del } \\
\text { sistema en la base de datos. }\end{array}$ \\
\hline $\begin{array}{l}\text { Seguimiento y } \\
\text { Control }\end{array}$ & $\begin{array}{l}\text { El seguimiento se realiza a través de internet, por medio de una URL que se conecta a la } \\
\text { Raspberry pi, que cuenta con un módulo Wireless LAN, el cual va conectado a los sensores. }\end{array}$ \\
\hline Página WEB & $\begin{array}{l}\text { La página WEB está desarrollada en un entorno PHP y HTML, adicionalmente se usaron scripts } \\
\text { de Java, CSS y algunas librerías graficas que ofrece Google como Google Charts. }\end{array}$ \\
\hline
\end{tabular}

Fuente: Elaboración con base a [14]. 
Tabla 3. Módulos del sistema propuesto por Maya Olalla y Chafla [16].

\begin{tabular}{|l|l|}
\hline \multicolumn{1}{|c|}{ Módulos } & \multicolumn{1}{c|}{ Descripción } \\
\hline $\begin{array}{l}\text { Recolección y } \\
\text { envío de datos }\end{array}$ & $\begin{array}{l}\text { Este módulo, hace uso de una red inalámbrica se sensores interconectados mediante una topología } \\
\text { tipo malla bajo el estándar 6loWPAN. Cada nodo cliente envía la información al nodo servidor } \\
\text { para el procesamiento de los datos; es de resaltar que una funcionalidad sobresaliente de este } \\
\text { tipo de topología de red es la posibilidad de retransmisión de información entre nodos contiguos. }\end{array}$ \\
\hline Servidor & $\begin{array}{l}\text { Servidor, permite el almacenamiento en una base de datos de las mediciones actuales de los } \\
\text { sensores de la red inalámbrica y dependiendo del estado de estos activa o desactiva el sistema } \\
\text { de riego por goteo en forma automática. }\end{array}$ \\
\hline $\begin{array}{l}\text { Control de } \\
\text { dispositivos }\end{array}$ & $\begin{array}{l}\text { Este módulo usa el sistema embebido MSP-CM5000, encargado de la recolección de datos } \\
\text { generados por los sensores del sistema, para su posterior procesamiento y almacenamiento; es } \\
\text { de resaltar que este módulo emplea el estándar 6loWPAN. }\end{array}$ \\
\hline $\begin{array}{l}\text { Seguimiento y } \\
\text { Control }\end{array}$ & $\begin{array}{l}\text { Red de sensores que permite la medición, transmisión, procesamiento y registros de los datos de } \\
\text { las variables como: temperatura y humedad relativa (sensor digital Sensirion SHT11), humedad } \\
\text { del suelo (YL-69 analógico), radiación solar (sensores Hamamatsu S1087 y S1087-01). }\end{array}$ \\
\hline $\begin{array}{l}\text { Aplicación } \\
\text { web }\end{array}$ & $\begin{array}{l}\text { Sitio web adaptable a cualquier dispositivo como PC, laptop, Tablet o teléfono inteligente, } \\
\text { presentando una interfaz gráfica al agricultor, con la información en tiempo real, permitiendo } \\
\text { el seguimiento y control de los dispositivos del riego. }\end{array}$ \\
\hline
\end{tabular}

Fuente: Elaboración con base a [14].

desarrollo ágil (Agile Software Development Cicle o $S L D C)[29,30]$. En cuanto al desarrollo del software se utilizó la metodología Scrum [31], llevando a cabo las siguientes actividades en forma secuencial:

Reunión equipo de trabajo-cliente. Se conocieron los puntos de vista del cliente, se formularon inquietudes y preguntas con el fin de tener una idea bastante clara del proyecto, resultados esperados y alcances, entre otros.

Reunión Scrum Máster y Product Owner. Se analizaron los objetivos del cliente, prioridades del proyecto y se definió el Sprint Backlog, junto con el conjunto de tareas, requerimientos y funcionalidades del proyecto. Una vez obtenido el Product Backlog, se planearon 4 Sprint de 4 semanas cada uno para el desarrollo del software.

Sprint Planning. Esta actividad se planificaba a inicio de semana, reuniendo el equipo de trabajo y el Product Owner. Como resultado, se obtenían las tareas a desarrollar y se asignaban a cada uno de los Account Managers.

Dayli Scrum. Diariamente, a la misma hora y lugar, los miembros del equipo se reunían y explicaban al Product Owner y Scrum Máster, las tareas realizadas durante el día, los problemas que se habían encontrado en el desarrollo de la actividad y las posibles soluciones a dichos problemas.
Sprint Review. Esta reunión se realizaba al final de cada Sprint involucrando a todo el equipo de trabajo. Se revisaban los requisitos cumplidos de acuerdo con el Sprint Planning; se realizaban pruebas de funcionamiento del producto obtenido con el fin de generar un feedback y en caso necesario hacer ajustes al Product Backlog para poder iniciar un nuevo sprint. Al final de los 4 Sprint proyectados se obtuvo una plataforma estable y con las funcionalidades proyectadas.

Respecto al desarrollo hardware e integración con el software, se tomó como referencia la metodología de modelo en $\mathrm{V}$ (procedimiento uniforme para el desarrollo de productos TIC y el perfeccionamiento de software embebido [26]). Con el fin de obtener resultados más confiables y de mayor calidad dentro de los límites de tiempo aceptables; en la etapa de validación, se trabajó bajo los parámetros establecidos por el estándar IEEE 1012-2016, el cual instaura el procedimiento para realizar la verificación y validación de sistemas, software y hardware [32], como se observa en la Figura 1.

\section{DISEÑO}

\section{Definición de especificaciones}

En esta fase se definieron y documentaron los requisitos del sistema, identificando las variables más significativas en cultivos, y analizando tecnologías similares. Así mismo se realizó la planificación 


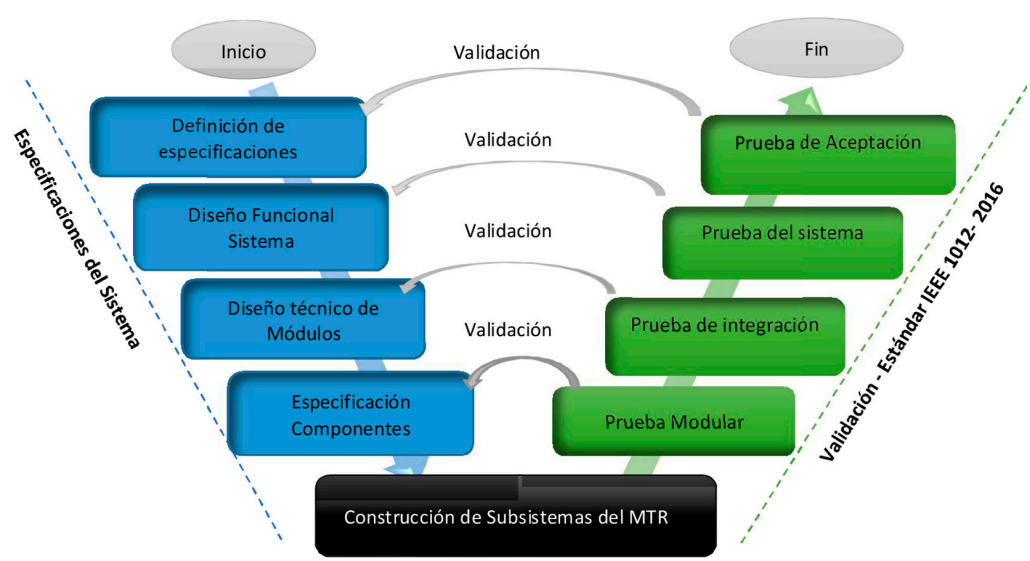

Figura 1. Metodología utilizada para el Desarrollo del Proyecto.

del proyecto, organizando el equipo de trabajo en cuatro grupos para el desarrollo de los subsistemas: hardware, comunicaciones, desarrollo de software web/móvil y un equipo para la validación e implementación del prototipo.

\section{Diseño funcional del sistema}

Se definió la arquitectura general del sistema, funcionalidades, especificaciones de firmware y software; así mismo se realizó un análisis de compatibilidad e interoperabilidad del sistema, evaluando variables y sensores, protocolos de comunicación, coste, tiempos, modularidad y confiabilidad del sistema, como se muestra en la Figura 2.

\section{Diseño técnico de los Módulos}

La arquitectura de AgriculTIC es modular y consta básicamente de tres subsistemas, los cuales se subdividen en diferentes unidades, como se describen a continuación:

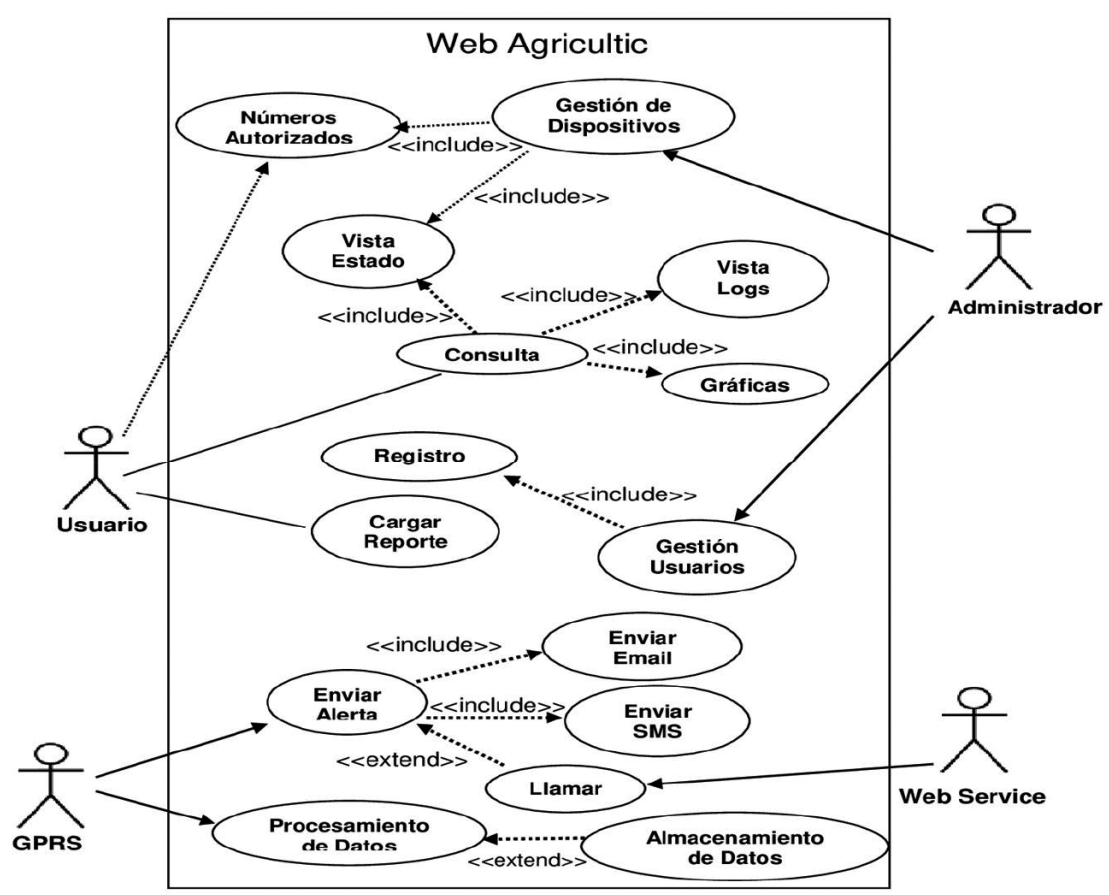

Figura 2. Diagrama de Caso de Uso de AgriculTIC. 
1. Subsistema hardware Terminal Remoto: compuesto por la CPU, encargada de registrar datos de los sensores, tomar decisiones y activar salidas de relé para los actuadores eléctricos, específicamente para los sistemas de riego. Sin embargo, puede activar otro tipo de actuadores como motores, sistemas de calefacción, sistemas de iluminación, etc.

2. Subsistema de comunicación: permite la conexión entre el Módulo Terminal Remoto y la plataforma web.

3. Subsistema de Aplicación para usuario final, (Plataforma web y aplicativo móvil para conexión en sitio).

\section{Especificación de Componentes}

Referente al hardware, se tuvo en cuenta criterios tales como: desempeño, facilidad de uso, disponibilidad de componentes en el mercado (Comercial-Off-The-Shelf: COTS), costos de implementación; además se seleccionaron como variables: la temperatura en grados centígrados $\left(\mathrm{T}^{\circ} \mathrm{C}\right)$, humedad del suelo y del aire (H.R \%), y $\mathrm{pH}$. En la Figura 3 se muestra un diagrama de componentes del sistema y en la tabla 1 los sensores utilizados.

\section{CONSTRUCCIÓN DE SUBSISTEMAS}

\section{Subsistema hardware terminal remoto}

La capa de hardware se compone de siete unidades: unidad central de procesamiento CPU; unidad de almacenamiento; unidad de medición de variables agrícolas; unidad de calendario y hora; unidad de comunicación local; unidad de activación de actuadores y unidad de comunicación con plataforma web. En la Figura 4 se aprecia un diagrama del subsistema en mención.

Se debe mencionar que uno de los criterios fundamentales en la selección de los componentes que conforman este subsistema, fue el bajo costo, buscando maximizar los resultados con una mínima inversión.

a) Unidad Central de Procesamiento CPU - Central Processing Unit.

En la Figura 5, se aprecia de forma general el algoritmo que ejecuta la CPU, quien es la encargada de registrar los datos de los sensores y recolectar la información en la unidad de almacenamiento interno. Por otra parte, establece la fecha y hora en la que se presentan los eventos,

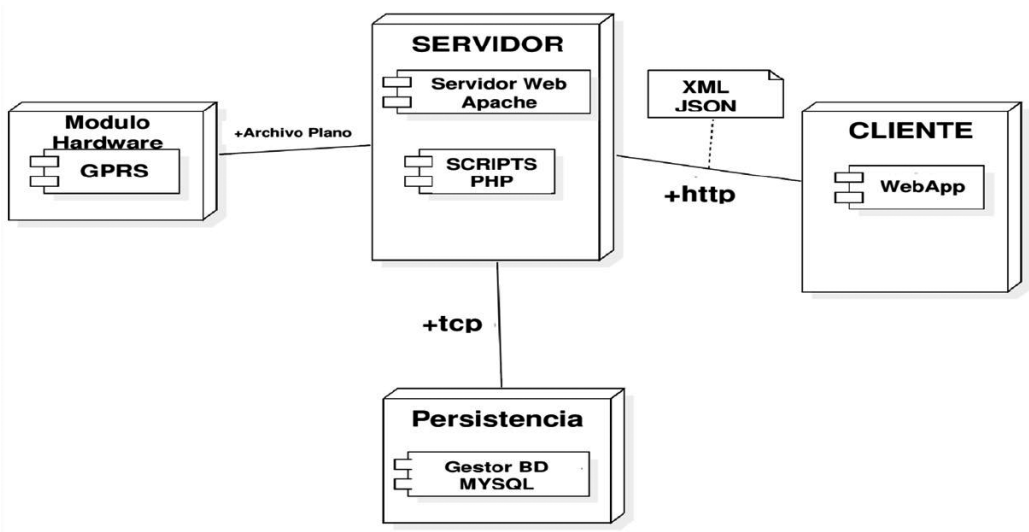

Figura 3. Diagrama de Componentes de AgriculTIC.

Tabla 4. Referencias de sensores utilizados para la validación del MTR

\begin{tabular}{|l|l|l|l|}
\hline \multicolumn{1}{|c|}{ Temperatura } & \multicolumn{1}{c|}{ Humedad del aire } & \multicolumn{1}{c|}{ Humedad del suelo } & \multicolumn{1}{c|}{ pH } \\
\hline $\begin{array}{l}\text { Ref. 18B20 de la empresa } \\
\text { Maxim Integrated }\end{array}$ & Ref. HS1101 de la empresa & Ref. CN1501 de la empresa & Ref. MSP430 \\
Humirel & ZHIPU & Sensor analógico \\
\hline
\end{tabular}

Fuente: Elaboración con base a sensores utilizados. 


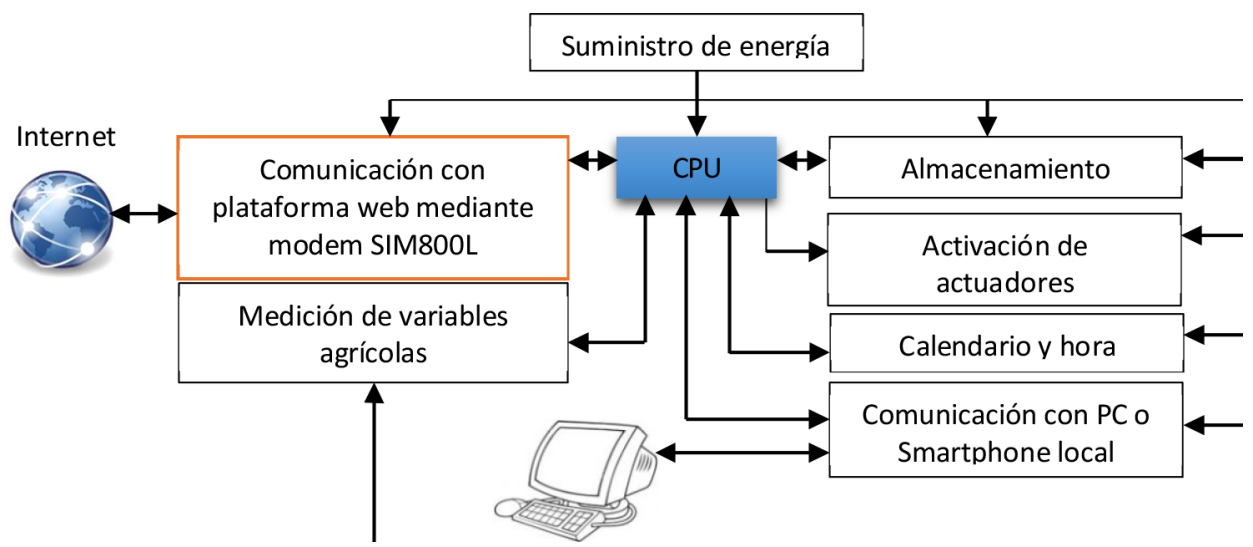

Figura 4. Diagrama de subsistemas que componen la capa de hardware.
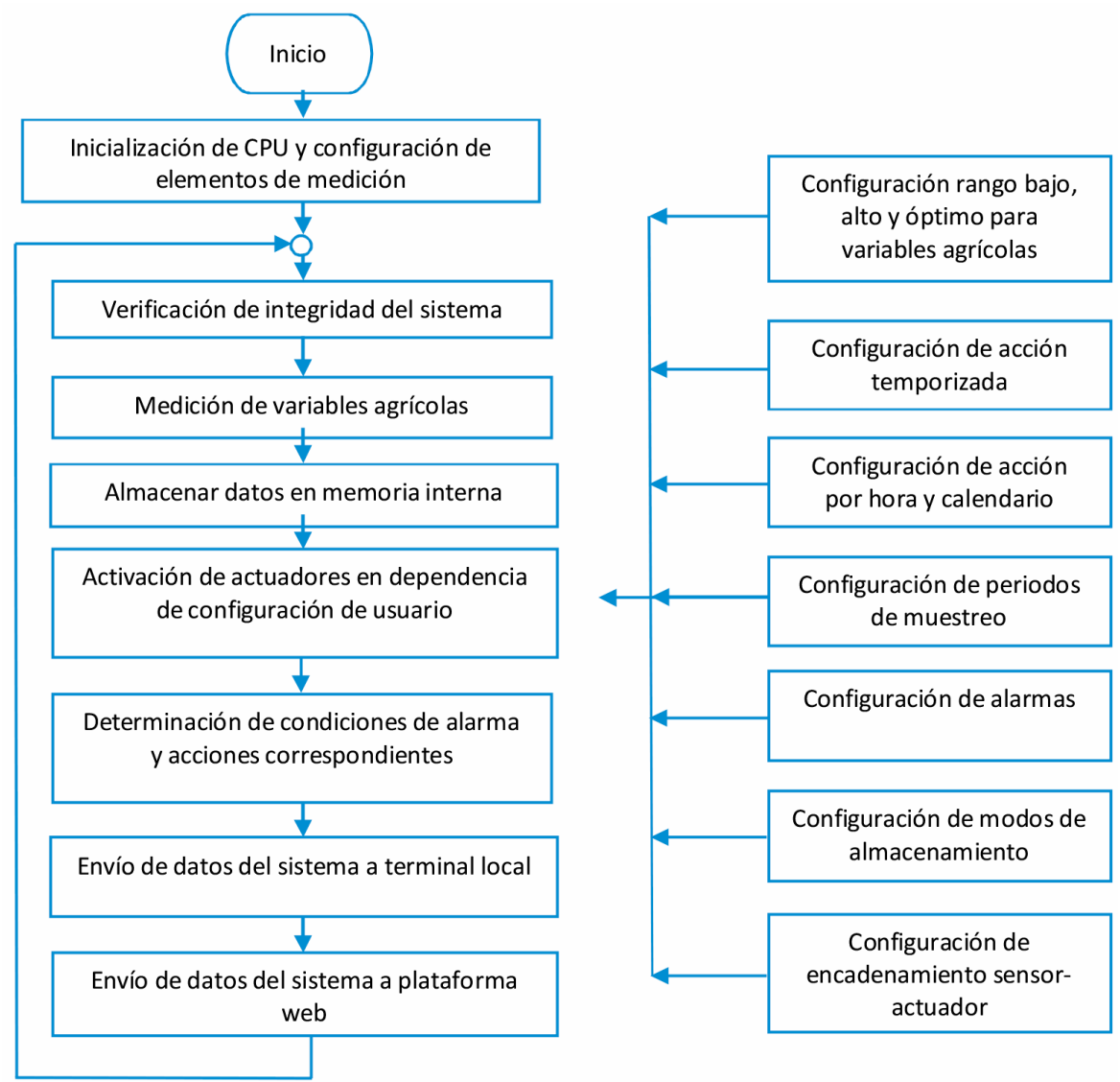

Figura 5. Algoritmo ejecutado por la CPU del sistema propuesto.

así mismo implementa las acciones que deben ejecutar los actuadores, y envía esta información a una base de datos en la nube a través de la conexión con la plataforma web. Este algoritmo es condicionado por el usuario; el cual puede programar alarmas, acciones, tiempos, secuencias de operación y otras configuraciones, accediendo desde una terminal virtual remota, o mediante una terminal local, que puede ser cualquier dispositivo con conexión USB OTG o Bluetooth. 
Para lograr los requerimientos mencionados se trabajó con el PIC18F4620 [33].

\section{b) Unidad de Almacenamiento}

Teniendo en cuenta que la memoria de datos del PIC18F4620 es insuficiente para el almacenamiento de datos, se implementó un módulo MSSP - Máster Synchronous Serial Port - configurado como bus I2C - Inter Integrated Circuit, para crear un bus que permitiera la inclusión de hasta $256 \mathrm{~KB}$ extras de memoria a través de dos dispositivos de almacenamiento AT24C1024; de este modo se contó con un total $321 \mathrm{~KB}$ de memoria no volátil de almacenamiento de datos.

c) Unidad de medición de variables agrícolas Inicialmente se propone la medición de seis variables: temperatura del aire, temperatura del suelo, humedad del aire, humedad del suelo, pH y Nivel de oxígeno en el agua; sin embargo, el sistema está abierto a infinidad de sensores que manejen señales de corriente, señales de voltaje, señales de ancho de pulso, señales de frecuencia entre muchas otras posibilidades.

- Temperatura. Sensor 18B20, entrega la medición en formato digital en un rango entre -55 y 125 grados centígrados [34].

- Humedad del aire. Sensor HS1101, tiene la capacidad de variar la capacitancia en dependencia de la humedad relativa presente en el ambiente, de acuerdo con la curva de transferencia del sensor [35].

- Humedad del suelo. Se debe mencionar que para el sensor CN1501, no se encuentra curva de transferencia suministrada por el fabricante, por esta razón, de forma empírica se tomaron los datos, y a partir del modelo propuesto por Rhoades "et al." [32], se construyó la curva de transferencia, en la cual se pudo determinar que el sensor tiene una variación no lineal de su resistencia eléctrica en función de la humedad del suelo; de esta forma, el hardware necesario para medir el valor de esta humedad, consta de un divisor de tensión, un resistor de $20 \mathrm{~K} \Omega$ y un dispositivo CN1501, siendo introducido el voltaje de este divisor a un conversor ADC de 10 bits, que hace parte de los periféricos que componen el PIC18f4620; asimismo, la CPU posee un algoritmo de linealización construido a partir de la curva de transferencia.
- $\quad$ pH: El medidor de $\mathrm{pH}$ analógico MSP430 cuenta con un conector (Bayonet Neill - Concelman) BNC, para una conexión instantánea de la sonda, obtiene mediciones de $\mathrm{pH}$ de $0-14$. Este sensor cuenta con un electrodo el cual debe ser calibrado con una solución tampón estándar para su funcionamiento.

\section{d) Unidad de calendario y hora}

El tiempo es una variable fundamental en los procesos agrícolas y está relacionado con todas las fases del proceso, pues es de gran importancia, determinar tiempos de aplicación de insumos, periodos de riego, estímulos al sistema, latencias varias, periodos de registro de datos, e infinidad de eventos que dependen del tiempo. En este contexto, es indispensable que el módulo de hardware cuente con un sistema que le permita ubicarse temporalmente; de esta forma, le fue añadido un reloj RTC - Real Time Clock- de la empresa Dallas semiconductor de referencia DS12887 [36]. Este es un dispositivo que cuenta con una batería de litio interna que le permite funcionar hasta por diez años sin alimentación externa, además cuenta con una memoria interna de 114 Bytes no volátil, NVRAM, debido a que los datos son mantenidos gracias a la batería interna, siendo posiciones de memoria de muy alta velocidad de escritura y de lectura, fundamentales para respaldar información de registros vitales en el funcionamiento del sistema.

e) Unidad de comunicación local con Smartphone $o$ PC.

Una característica de la capa hardware, es que está en capacidad de ser programada de forma local, para esto, se dispuso de dos puertos; uno USB y otro Bluetooth. El puerto USB hace posible que el procesador de la capa de hardware intercambie datos con una aplicación mediante la UART del PIC18F4620; para alcanzar este propósito, se usa el conversor, FT232RL de la empresa "Future Technology Devices International" el cual convierte datos entre el protocolo USB y niveles TTL de RS232. Gracias a esta tecnología, es posible que a través de un smartphone con soporte OTG, se establezca una comunicación transparente para realizar procesos de lectura y configuración del MTR. Adicionalmente, el dispositivo, posee un 
puerto de comunicación Bluetooth mediante el dispositivo HC-06 de la empresa "Guangzhou HC Information Technology".

\section{f) Unidad de activación de actuadores}

Esta unidad dispone de cuatro salidas digitales que pueden ser configuradas de modo "ONOFF" o como salidas PWM; adicionalmente, cuenta con cuatro salidas tipo relé, con una capacidad de manejo de corriente de hasta 30 amperios; con una bornera de tres contactos por cada uno de los relés ; un contacto común, uno normalmente abierto y otro normalmente cerrado que pueden ser conectados en dependencia de las circunstancias particulares en que el sistema sea empleado; estas salidas son configurables y programables mediante el desarrollo logrado en la capa de software; en la Figura 6 se aprecia una imagen del aspecto de la tarjeta final desarrollada.

\section{Subsistema de comunicación del MTR con el servicio web}

En esta sección se describe el proceso que se llevó a cabo para establecer la comunicación entre el Módulo Terminal Remoto y la plataforma web. Este subsistema está integrado principalmente por dos elementos: Un Modem SIM800L y un Microcontrolador PIC18F252.

\section{a) Módulo SIM800L}

El modem SIM800L, es un dispositivo GSM/ GPRS Quad-Band que trabaja con frecuencias de 850/900/1800/1900 MHz. Este módulo permite generar llamadas de voz y enviar y recibir mensajes de texto; funciones que son necesarias a la hora de implementar el sistema de alertas generadas por los cultivos. Por otra parte, y una de las principales razones por la cuales se trabajó con este dispositivo, es que soporta protocolos de transmisión de internet como, HTPP, TCP/IP y FTP, lo que permite hacer peticiones tipo GET o POST, evitando así el uso de un servidor VPS o dedicado, lo cual incrementarían notablemente los costos de operación del sistema. En la Figura 7 se muestra la tarjeta desarrollada.

Esta tarjeta es controlada por la CPU a través del puerto UART, haciendo uso de comandos AT habilitados para el SIM800L. Para tener acceso a la red GSM, se requiere de una Sim card, registrada en un operador de telefonía celular; posteriormente la CPU envía de forma secuencial los comandos AT, permitiendo la conexión con el servicio GPRS para la transmisión y recepción de datos a través de internet móvil; igualmente abre las sesiones HTPP, las cuales permiten conectarse a la URL habilitada en el servidor, para registrar los datos de sensores, actuadores y estado general del MTR y finalmente lee la configuración realizada por el usuario desde la plataforma web.

b) Módulo microcontrolador PIC18f252

Este microcontrolador es el cerebro del módulo ya que mantiene una comunicación full dúplex con el modem SIM800L y el hardware de los sensores. Dentro de sus principales funciones están: configurar y gestionar el Modem SIM800L; configurar el servicio HTTP, realizar las configuraciones del módulo de sensores de acuerdo con los parámetros establecidos por el usuario y recibir y procesar la trama de configuración desde el servidor web. La

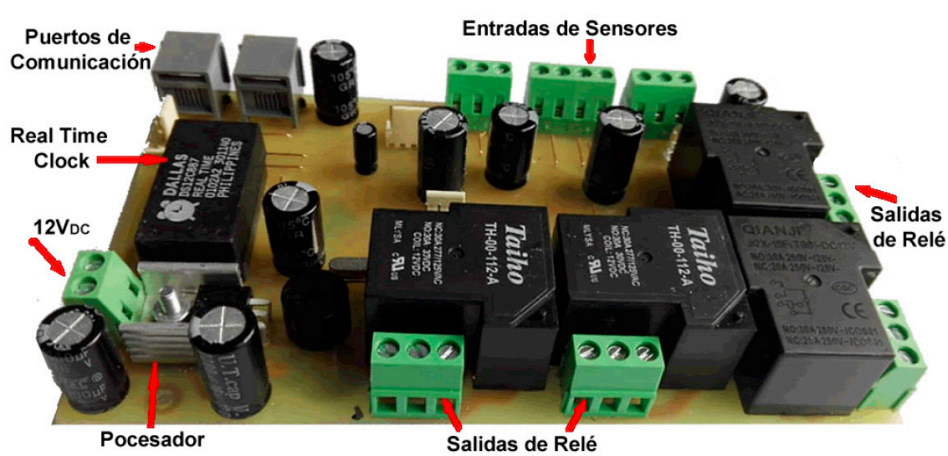

Figura 6. Aspecto de tarjeta CPU, relés, reloj, almacenamiento. 


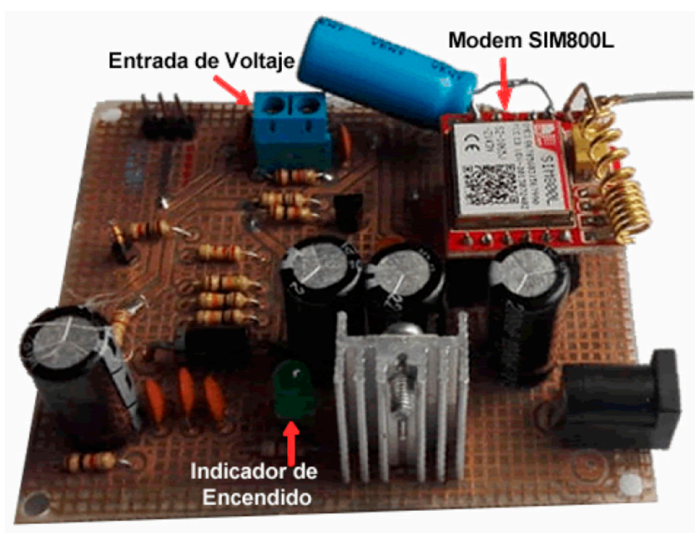

Figura 7. Tarjeta diseñada para el Módulo SIM800L.

implementación se realizó a través del circuito que se muestra en la Figura 8, el cual integra los puertos de conexión hacia el modem SIM800L y el módulo de sensores; además contiene la memoria de almacenamiento de la información de configuración y los indicadores visuales, entre otros.

Una de las funcionalidades más importantes de este circuito, es el almacenamiento de los datos registrados por los usuarios, como, ID asignado al dispositivo; número de celular para envío de alarmas; comandos de ejecución; configuración del dispositivo; horarios de activación/desactivación de actuadores; usuarios y claves de acceso al servidor web; valores máximos y mínimos de sensores; acciones sobre los relés y programación o agendamiento de Relés (Fechas y Horas). En este sentido se integró al módulo, una memoria EEPROM 24LC256, la cual permite el almacenamiento de hasta 256 Kilobytes de datos.

\section{Subsistema de Aplicación para usuario final, WebAPP}

La aplicación web permite que los usuarios del sistema gestionen los dispositivos asignados, el dominio de trabajo es http://agricultic.com. Entre las funcionalidades del sistema se encuentran:

a) Gestión de Usuarios (Rol Administrador) Para el proceso de autenticación se debe usar el correo y la contraseña asignada al usuario. En la Figura 9 se observa una vista del rol Admin. Admin $\rightarrow$ Rol que permite al usuario realizar tareas administrativas como gestionar otros usuarios, otros dispositivos, tipos de sensores y relés.

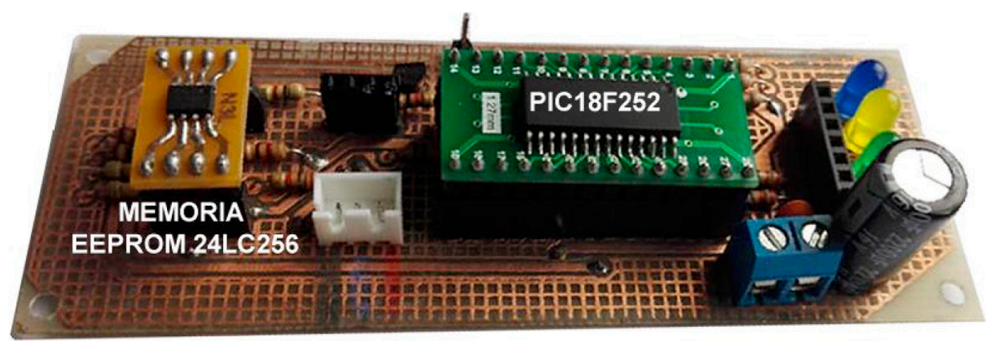

Figura 8. Circuito desarrollado con el PIC18F252.

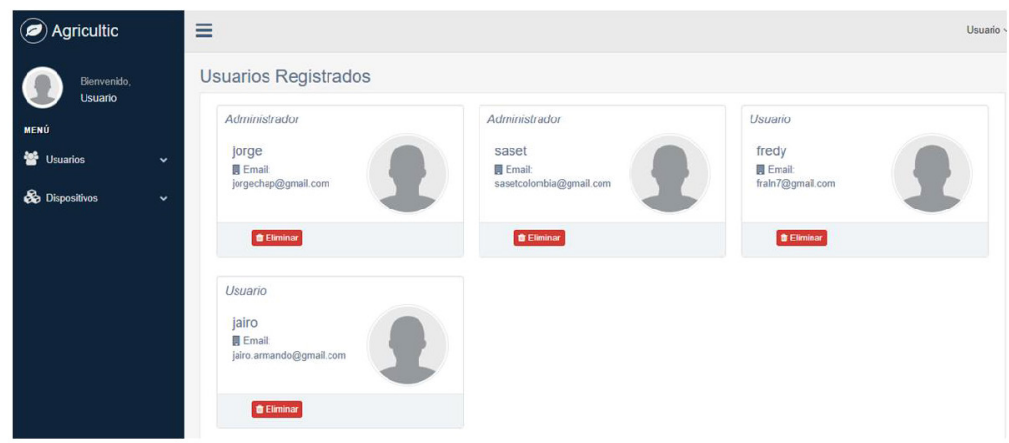

Figura 9. Vista gestión de usuarios. 
User $\rightarrow$ Rol que usa los dispositivos asignados por el Admin y los puede gestionar en forma limitada. Obtiene informes, visualiza estado y comportamiento de los sensores en tiempo real.

b) Gestión de Dispositivos. El administrador crea dispositivos con sus respectivos sensores y relés, el usuario por su parte gestiona los relés y sensores. El usuario del sistema asigna números de teléfono a los dispositivos para recibir las alertas respectivas.

El dispositivo envía los valores de los sensores al sistema a través de una petición a una URL con el método GET. El sistema los recibe, analiza y almacena, de ser necesario envía alertas a través de correo electrónico indicando la novedad del sensor.

c) Gestión de Relés. El usuario del sistema asigna acciones a cada relé para que se prenda o apague cuando el valor de un sensor exceda o esté por debajo de nos límites establecidos. Igualmente, para cada relé se puede programar o agendar que prenda o apague determinado día, la hora de inicio, la duración y la frecuencia o repeticiones de la acción.

d) Gestión de Sensores. El usuario del sistema modifica, los parámetros o valores de los sensores para ajustarlos a sus necesidades del cultivo como se observa en la Figura 10.

e) Envío de Alertas. El dispositivo envía alertas a través de llamada telefónica, un mensaje de texto (SMS) o un correo electrónico. Lo anterior cuando los valores de los sensores se exceden o bajan de un rango especificado.

f) Estado de los sensores. A través de la aplicación se visualiza en tiempo real, el estado de los sensores o valor actual a través de un componente visual como se observa en la Figura 11.
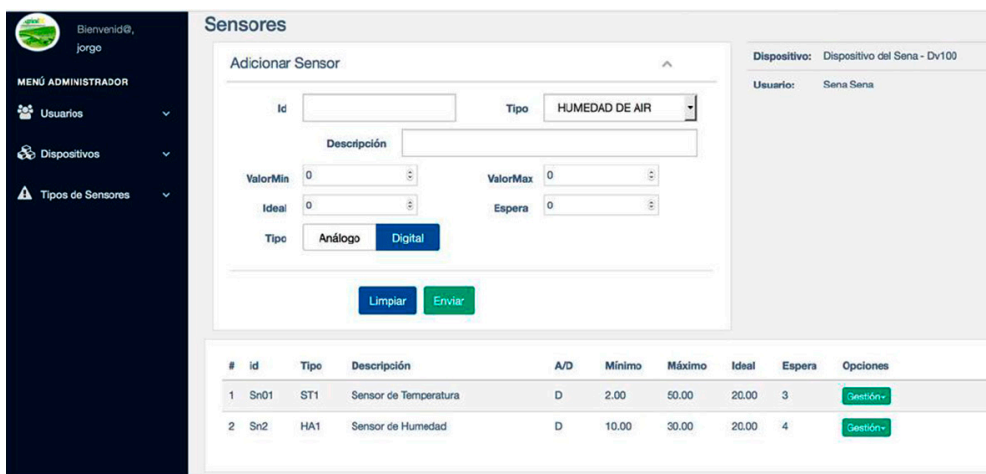

Figura 10. Vista gestión de sensores.
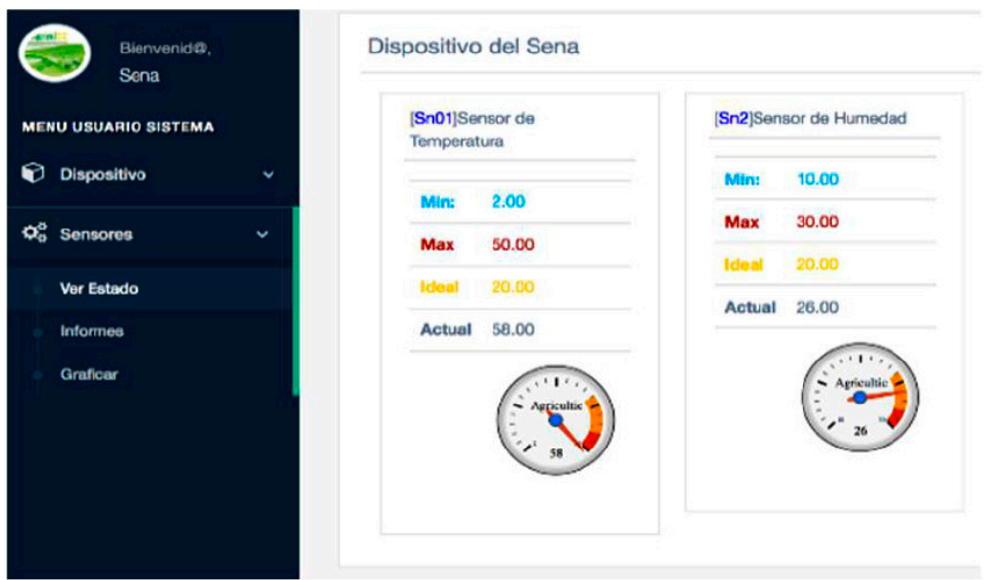

Figura 11. Vista estado de los sensores. 
g) Reportes. Se visualiza el historial de los valores de los sensores que el dispositivo ha enviado al sistema, se pueden exportar los valores en diferentes formatos.

h) Graficar. A través de gráficos de líneas se observa el comportamiento para un sensor determinado dentro de un rango de tiempo especificado, como se muestra en la Figura 12.

\section{VALIDACIÓN Y PRUEBAS DEL SISTEMA}

Las pruebas del sistema se realizaron según la Norma IEEE 1012- 2016, la cual establece que los procesos de verificación y validación $(\mathrm{V} \& \mathrm{~V})$ se utilizan para determinar si los productos de desarrollo de una actividad determinada cumplen con los requisitos y si el producto satisface su uso previsto y las necesidades del usuario [32]. Esta norma se aplica a los sistemas, software y hardware que se desarrollan, utilizando componentes disponibles en el mercado Comercial Off-The-Shelf (COTS). A continuación, en la Figura 13 se describen las principales pruebas según el estándar IEEE 10122016 que fueron utilizadas para el desarrollo del hardware del proyecto. a) Prueba modular: "consiste en la prueba de cada módulo aislado del resto del sistema". Cada grupo de trabajo por separado realizó las pruebas de los distintos subsistemas, validando su correcto funcionamiento y revisando la interoperabilidad.

- Subsistema hardware: se realizaron pruebas de la CPU, recepción de información de variables de los sensores, calibración de transductores, pruebas de control y funcionamiento de actuadores, pruebas de adaptación de señales, pruebas de capacidad de memoria, pruebas del reloj del sistema y pruebas de programación y configuración básica en sitio.

- Subsistema de comunicación: se realizaron pruebas de configuración de protocolos, recepción y transmisión de datos de sensores, conexión con el servidor web, pruebas del microcontrolador que mantiene la comunicación full dúplex con el Modem SIM800L, pruebas de control y almacenamiento de información de usuarios, pruebas de transparencia de datos referente a los formatos de datos utilizados por los protocolos.

- Plataforma web se realizaron pruebas de funcionalidad de los módulos, pruebas de bases
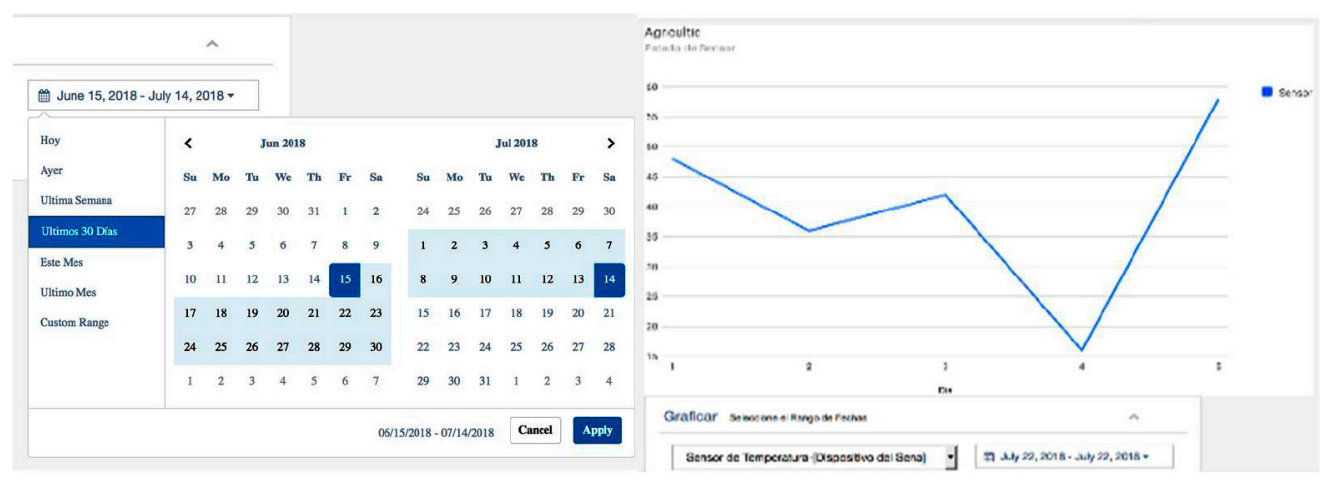

Figura 12. Vista del módulo Graficar.

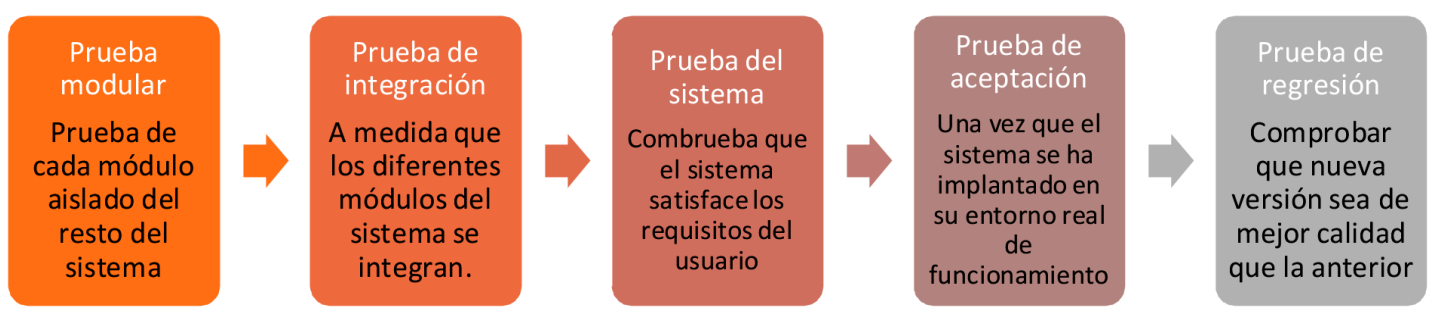

Figura 13. Pruebas realizadas según el estándar IEEE 1012-2016. 
de datos, pruebas de formularios y registros de información, intercambian datos a través de XML y JSON; pruebas de integración de bases de datos con formularios de registros y pruebas de seguridad del sistema.

b) Prueba de integración: "se realiza a medida que los diferentes módulos del sistema se integran". El objetivo fundamental de esta prueba es comprobar que las interfaces entre los distintos módulos son correctas. En esta etapa se realizó la integración de los tres subsistemas, siendo fundamental el módulo de comunicación el cual recibe los datos del módulo de sensores, y adapta las tramas nuevamente para retransmitirlos a la URL. En cuanto a la integración física de las tarjetas se diseñó una caja modular para asegurarlas y dejar expuestos los distintos conectores, tal como se observa en la Figura 14.

c) Prueba del sistema: "se realiza cuando se han integrado todos los módulos"; su objetivo es

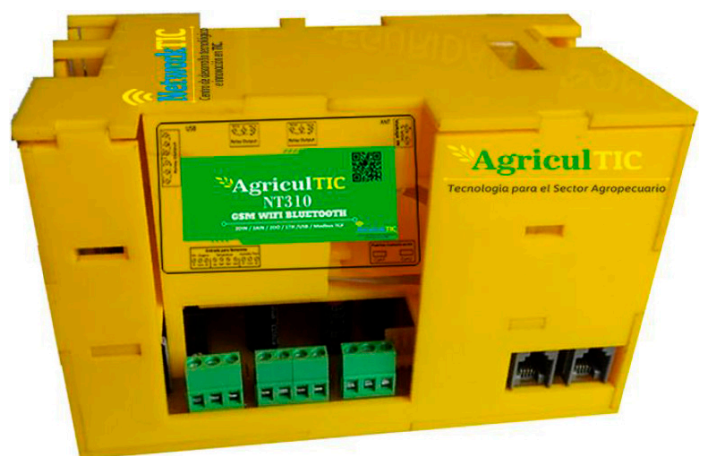

Figura 14. Vista frontal del prototipo final del MTR, AgriculTIC. comprobar que el sistema satisface los requisitos del usuario, tanto los funcionales como los no funcionales.

En este sentido se elaboraron tres prototipos similares del MTR, uno se dejó en el laboratorio y los otros dos se llevaron a un cultivo de piña y hongo de Orellana, establecidos en la granja experimental el Remanso, de la Universidad Unitrópico, ubicada en Yopal Casanare Colombia. Respecto a la piña se estableció un cultivo demostrativo variedad MD2 en un espacio de $20 \mathrm{~m}^{2}$, el cual se instrumentó con los sensores de humedad del suelo y del aire, temperatura y $\mathrm{pH}$, como se observa en la Figura 15. Como actuadores se utilizaron dos bombas de $1 / 2 \mathrm{HP}$, las cuales alimentaron un sistema de riego por aspersión. La alimentación del módulo se realizó a través de un panel solar a fin de mantener constante el flujo de energía.

El cultivo de hongo de Orellana se estableció en un espacio cerrado, el cual fue instrumentado con los sensores validados de humedad relativa, temperatura y $\mathrm{pH}$; y como actuador se utilizó una electrobomba de $1 / 2 \mathrm{HP}$, la cual suministro el agua al sistema de riego por nebulización. El control se realizó a través del MTR, como se observa en la Figura 16.

d) Prueba de aceptación: "se realiza una vez que el sistema se ha implantado en su entorno real de funcionamiento"; su objetivo es demostrar al usuario que el sistema satisface sus necesidades. Las pruebas de aceptación finales del prototipo se llevaron a cabo inicialmente por el Coordinador del proyecto de la empresa NetworkTIC S.A.S, posteriormente, fue aceptado por la entidad encargada de la revisión del convenio, en este

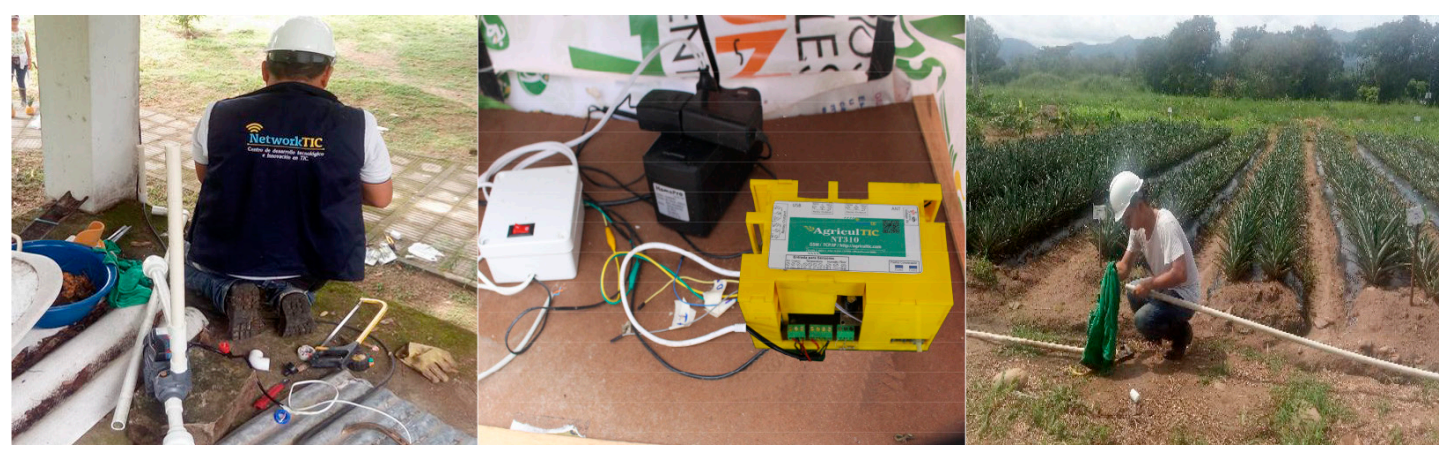

Figura 15. Pruebas de funcionamiento en cultivo de Piña. 

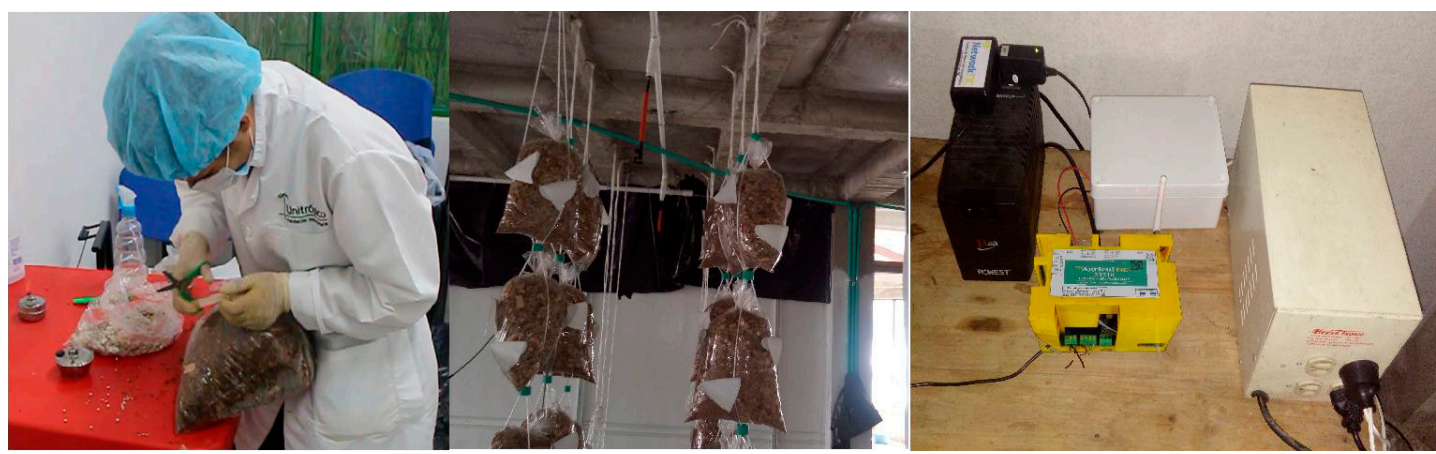

Figura 16. Pruebas de funcionamiento en cultivo de Orellana.

caso la Universidad Abierta y a Distancia UNAD y finalmente fue aprobado por SENNOVASENA, regional Casanare, quien a través de la convocatoria pública nacional "fomento IDT 2015-2017- Mipymes Proceso 1", cofinanciaron el proyecto.

e) Prueba de regresión: tiene como objetivo comprobar que toda nueva versión de un producto software es de no menos calidad que la versión anterior, es decir, que al introducir cambios no se ha reducido la valoración de ninguna de las características de calidad que tenía el producto. Esta prueba no aplica para este caso dado que es una primera versión.

\section{RESULTADOS FINALES}

\section{Pruebas}

Como se describió en la sección de pruebas, los tres subsistemas (unidad central de procesamiento, módulo de comunicación y plataforma web), funcionaron eficientemente a nivel individual y en la etapa de integración. Respecto a los sensores seleccionados se ajustaron a las necesidades requeridas, dado que permitieron capturar las variables ambientales y se logró la transmisión a internet sin ningún contratiempo.

Por otro lado, los materiales utilizados en este desarrollo son de fácil consecución en el mercado y muy económicos.

Respecto a la aplicación web, se debe mencionar que esta funcionó correctamente y se logró comunicar de forma aceptable con el hardware desarrollado; permitiendo observar en tiempo real los datos obtenidos a partir de los sensores; se debe destacar la buena aceptación de la interfaz gráfica de usuario, amigable y entendible; gracias al diseño "responsive" que se adapta a diferentes plataformas digitales disponibles en la actualidad.

El trabajo en campo y la validación en dos cultivos diferentes, permitió ajustar el MTR de forma tal que se minimizo la posibilidad de fallos, gracias a las diferentes pruebas en campo; de esta forma se garantizó la fiabilidad y robustez del sistema.

Adicionalmente, se realizó un análisis integral de los resultados obtenidos del funcionamiento del hardware, del software y de los sensores validados; comprobando que se cumplió a cabalidad con el objetivo planteado para este proyecto, tal como se puede verificar en la carta entregada por SENNOVA del Servicio Nacional de Aprendizaje SENA, quien es la entidad financiadora del proyecto.

\section{Comparativas}

A fin de comparar el Módulo Terminal Remoto MTR, descrito en este artículo, con los sistemas vistos en la sección de Antecedentes; Trabajos Relacionados, se definieron dos enfoques. Un enfoque técnico, referente a características tecnológicas de protocolos, herramientas y dispositivos utilizados en la materialización de estos mecanismos y el otro enfoque asociado a los costos de fabricación y disponibilidad de componentes en el mercado.

\section{Factor tecnológico}

Respecto al factor tecnológico comparativo se trabajó con: sensores, microcontroladores, tipo de conexión a internet, mecanismos de comunicación, reloj de tiempo real (RTC), protocolos abiertos, 
almacenamiento de información, salidas de relé, plataforma web y alcance de la red. En la Tabla 5 se muestran los resultados de esta comparación.

Como se puede observar en la tabla 5, el módulo MTR AgriculTIC posee ciertas ventajas frente a los dispositivos relacionados. En los tres trabajos revisados, utilizan hardware comercial (ARDUINO, RASPBERRY PI 3 y MTM-CM5000-MSP), esto limita las posibilidades y minimiza una posible patente o comercialización, mientras que AgriculTIC, desarrolló un hardware propio, por lo que no utiliza

Tabla 5. Comparación tecnológica del MTR AgriculTIC Vs Trabajos Relacionados.

\begin{tabular}{|c|c|c|c|c|}
\hline Factor comparativo & $\begin{array}{c}\text { Trabajo 1. Sistema web } \\
\text { de bajo costo [11] }\end{array}$ & $\begin{array}{l}\text { Trabajo 2. Puentes. } \\
\text { Jonathan }[14]\end{array}$ & $\begin{array}{l}\text { Trabajo 3. E. A. Maya } \\
\text { Olalla, [16]. }\end{array}$ & $\begin{array}{l}\text { MTR AgriculTIC } \\
\text { Sistema Propuesto. }\end{array}$ \\
\hline Microcontrolador & NodeMCU esp8266 & PIC18F4550. & $\begin{array}{l}\text { Microcontrolador } \\
\text { MSP430F1611. }\end{array}$ & PIC18F4620 у PIC18F252. \\
\hline Sensores & $\begin{array}{l}\text { AM2301, Sht10, MQ135, } \\
\text { BH1750 }\end{array}$ & $\begin{array}{l}\text { D H T } 22 \text { / A M } 2302, \\
\text { BH1750FVI. }\end{array}$ & $\begin{array}{l}\text { H.R y T SHT11. Lumino- } \\
\text { sidad S1087 y Humedad } \\
\text { YL-69 Lluvia LY-83. }\end{array}$ & $\begin{array}{l}\text { 18B20, HS1101, CN1501, } \\
\text { MSP430. }\end{array}$ \\
\hline $\begin{array}{l}\text { Mecanismos de } \\
\text { comunicación }\end{array}$ & $\begin{array}{l}\text { La placa NodeMcu se co- } \\
\text { munica en forma inalám- } \\
\text { brica y directa con otras } \\
\text { placas y con el servidor } \\
\text { en internet. }\end{array}$ & $\begin{array}{l}4 \text { puertos USB 2.0, un } \\
\text { puerto ETHERNET, una } \\
\text { salida de video Full HDMI, } \\
\text { módulo Wireless LAN. }\end{array}$ & $\begin{array}{l}\text { Estándar 6loWPAN, antena } \\
\text { CC2420 de Texas Instruments } \\
\text { y un border-router. } \\
\text { Usa comunicación serial } \\
\text { - UART. }\end{array}$ & $\begin{array}{l}\text { Soporta Puertos USB OTG, } \\
\text { RS232, Bluetooth. } \\
\text { Protocolos TCP/IP. } \\
\text { GPRS, 3G y 4G. }\end{array}$ \\
\hline $\begin{array}{l}\text { Almacenamiento de } \\
\text { información }\end{array}$ & $\begin{array}{l}\text { Placas NodeMCU, basadas } \\
\text { en el módulo Esp8266. En } \\
\text { la nube en el servidor }\end{array}$ & $\begin{array}{l}\text { Raspberry pi } 3 \text { cuenta con } \\
\text { memoria RAM de } 1 \mathrm{~GB} .\end{array}$ & $\begin{array}{l}\text { Placa MTM - CM5000 } \\
\text { - MSP. } \\
\text { Memoria 1MB External } \\
\text { Flash. }\end{array}$ & $\begin{array}{l}\text { Local: } 321 \mathrm{~KB} \text { de memoria } \\
\text { no volátil + } 256 \mathrm{~KB} \text { de la } \\
\text { EEPROM. En la nube en el } \\
\text { servidor. }\end{array}$ \\
\hline Alcance de la red & $\begin{array}{l}\text { Alcance es amplio y el } \\
\text { servidor está en internet }\end{array}$ & $\begin{array}{l}\text { Dominio público, servidor } \\
\text { está en internet. }\end{array}$ & $\begin{array}{l}\text { RLP(IPv6). Dominio público, } \\
\text { servidor está en internet. }\end{array}$ & $\begin{array}{l}\text { Servidor está en internet. } \\
\text { Además, utiliza GPRS, } 3 G \\
\text { y } 4 \text { G para texto y voz. }\end{array}$ \\
\hline Salidas de Relé & 2 & NO & 2 & 4 \\
\hline Plataforma Web & $\begin{array}{l}\text { Framework de apli- } \\
\text { caciones web PHP y Twig, } \\
\text { (MVC), Java-Script, css y } \\
\text { SQL para la base de datos. }\end{array}$ & $\begin{array}{l}\text { PHP y HTML, scripts } \\
\text { de Java, CSS y algunas } \\
\text { librerías graficas que ofrece } \\
\text { Google como Google } \\
\text { Charts. }\end{array}$ & $\begin{array}{l}\text { Sitio web adaptable a cual- } \\
\text { quier dispositivo como PC, } \\
\text { laptop, Tablet o teléfono } \\
\text { inteligente. }\end{array}$ & $\begin{array}{l}\text { Permite alos usuarios gestionar } \\
\text { los dispositivos y generar } \\
\text { informes del estado de los } \\
\text { sensores. Gestiona Relés, } \\
\text { Envía Alertas. } \\
\end{array}$ \\
\hline Reloj de tiempo real (RTC) & NO & SI. RTC DS1307 & SI & SI. RTC DS12887 \\
\hline $\begin{array}{l}\text { Protocolos abiertos de } \\
\text { comunicación }\end{array}$ & NO & NO & NO & SI \\
\hline Fuente de Alimentación & $\begin{array}{l}\text { Cargador solar para celular } \\
\text { que sirve para alimentar el } \\
\text { Arduino o AC230V. }\end{array}$ & $\begin{array}{l}\text { Micro USB socket } 5 \mathrm{~V} 1 \text {, } \\
2.5 \mathrm{~A} \text {. }\end{array}$ & $\begin{array}{l}\text { 3V (2xAA Battery Holder } \\
\text { Provided). }\end{array}$ & $\begin{array}{l}\text { Adaptador Estándar de 12V } \\
\text { DC; } 1 \text { Amperio. }\end{array}$ \\
\hline $\begin{array}{l}\text { Uso de placas ARDUINO o } \\
\text { similares }\end{array}$ & $\begin{array}{l}\text { SI. Utiliza placa Node- } \\
\text { MCU Arduino. }\end{array}$ & $\begin{array}{l}\text { SI. Utiliza la Raspberry } \\
\text { pi } 3 .\end{array}$ & SI. MTM-CM5000-MSP. & NO. El desarrollo es propio. \\
\hline
\end{tabular}

Fuente: Elaboración con base a [11, 14 y 16] y MTR AgriculTIC.

Tabla 6. Costos de dispositivos utilizados en la construcción (US\$).

\begin{tabular}{|c|c|c|c|c|c|}
\hline \multirow{2}{*}{$\begin{array}{c}\text { Trabajo } \\
\text { Relacionado }\end{array}$} & \multicolumn{4}{|c|}{ Tecnologías Utilizadas } & \multirow{2}{*}{$\begin{array}{c}\text { Costo } \\
\text { total }\end{array}$} \\
\cline { 2 - 5 } & Microcontrolador & Placas base & Sensores & Comunicación & \\
\hline$[11]$ & 13,69 & 7,65 & 65,14 & 0 & 92,52 \\
\hline$[12]$ & 8,64 & 96,03 & 40,00 & 40,00 & 184,67 \\
\hline$[16]$ & 19,21 & 95,00 & 41,61 & 27,00 & 182,82 \\
\hline AgriculTIC & 16,00 & 0 & 58,26 & 32,01 & 106,27 \\
\hline
\end{tabular}

Fuente: Elaboración con base a [11, 14 y 16] y MTR AgriculTIC. 
ni depende de placas comerciales. Adicionalmente, se debe mencionar que AgriculTIC cuenta con canales de comunicación, alternos a internet, como: comunicación GPRS, 3G y 4G para mensajes de texto y voz; estas características favorecen su uso en zonas en donde no existe acceso a internet. Por otra parte, este proyecto deja los protocolos de comunicación abiertos, dando la posibilidad a otros desarrolladores para que utilicen este hardware en múltiples aplicaciones, permitiendo particularizar su uso.

\section{Costos}

En la Tabla 6 se muestra los costos aproximados de los dispositivos utilizados en cada uno de los trabajos relacionados y del trabajo propuesto, este último al final de la tabla. Cabe anotar que los precios de los componentes son precios del mercado buscados en plataformas comerciales de internet a fecha de enero de 2020.

Como se puede observar en la Tabla 6, el trabajo propuesto frente a los demás, presenta un costo más bajo excepto el desarrollado por M. Mamani, M. Villalobos, and R. Herrera [11], que es un sistema web de bajo costo, con una diferencia de 14 dólares aproximadamente. Sin embargo, este proyecto trabaja con una placa Nodemcu esp8266, lo cual limita su posibilidad de patentamiento al no ser hardware propietario. Finalmente, en este sentido, se debe mencionar que, tomado como referencia lo mencionado en [13, 15], el precio de AgriculTIC es razonable y accesible, teniendo un costo aproximado del $42,45 \%$ del costo de los equipos del mercado de este tipo, tomados como referencia, haciendolo un producto competitivo en términos económicos $\mathrm{y}$ evidentemente en términos prestacionales.

\section{Confiabilidad del sistema}

Un factor muy importante para este sistema es la confiabilidad. En este sentido se optimizó la Generación de Alertas programables, tanto por correo electrónico mensaje de texto o llamada. Además de las alarmas el sistema cuenta con realimentación del estado real de las salidas de relé, lo cual permite un monitoreo efectivo del estado real de los actuadores.

Otras posibles Aplicaciones del MTR AgriculTIC Aunque el MTR AgriculTIC, fue desarrollado para el sector agropecuario; este sistema puede operar con muchos otros sensores siempre y cuando soporten señales eléctricas de voltaje, corriente o pulsos electromagnéticos. Por otra parte, el protocolo de comunicación es abierto dándole la oportunidad a otros desarrolladores de adaptar el módulo para diversas aplicaciones dentro de las que se pueden mencionar: sistema de monitoreo automático, sistemas de alarma de seguridad, estaciones de bombeo, control remoto de estaciones meteorológicas y registro de datos, control de válvulas eléctricas, ahorro de energía, sistema de control de luces de edificios y calles, Control y monitoreo de tanques de almacenamiento de líquidos y sistemas de control de acceso entre otros.

\section{CONCLUSIONES}

El MTR emite cada segundo por el puerto serial RS232, una trama de datos, con información del estado de los sensores, relés, contadores y configuraciones. Por otra parte, cuenta con un puerto independiente para recepción de comandos de configuración estandarizado, lo cual le permite generar un protocolo de comunicación abierto. Esta característica brinda la posibilidad a otros desarrolladores para generar nuevas soluciones e incorporar diferentes alternativas de comunicación; siendo esta una innovación que abre infinitas posibilidades para crear diversos productos tecnológicos enfocados en telemetría, telemando, telecontrol y agrometría.

El Internet de las cosas - (IoT), es un concepto que hace posible que dispositivos cotidianos o de índole industrial se conecten a la red, permitiendo la gestión remota de estos y el monitoreo permanentemente del estado de funcionamiento, así como también indicando condiciones críticas o de alarma para ser atendidas de forma prioritaria. A partir de este concepto, es posible construir una capa de hardware, adaptada a las necesidades y circunstancias particulares de la producción agrícola del país, permitiendo aplicar a esta industria, la diversidad de adelantos tecnológicos contemporáneos en el campo de las comunicaciones y la informática.

El trabajo en campo muestra que la detección temprana, el seguimiento y acción sobre un proceso agrícola, tiene una fuerte dependencia de variables que pueden ser medidas en el terreno, como la humedad, la temperatura, la iluminación y el pH entre muchas otras; sin embargo, también inciden otras variables que no pueden ser medidas directamente y deben 
ser inferidas, tales como periodos de tiempo entre acciones sobre los cultivos, latencias, estimaciones sobre variables climáticas, sobre la aplicación de insumos etc. En estos casos es relevante el uso de registros digitales, del procesamiento de datos y de la generación de informes que determinan las acciones a seguir en el proceso de forma automática o asistida. En este aspecto, la capacidad de almacenamiento y procesamiento de datos, aunado al seguimiento y la capacidad de acción a distancia, son evidentemente una solución viable; razón por la cual el presente trabajo busca sacar ventaja de estas tecnologías contribuyendo a su aplicación en la industria agrícola del país.

El módulo Terminal Remoto, permite que el usuario pueda realizar las respectivas configuraciones o lecturas de estado general, mediante el envío de mensajes de texto (SMS), incluyendo al comienzo del mensaje el ID asignado al dispositivo y la clave de usuario. Como respuesta el módulo devolverá un SMS con la información solicitada (por ejemplo, estado de sensores y/o actuadores) o la confirmación de la acción.

La inclusión del reloj de tiempo real (Real Time ClockRTC), es fundamental para determinar de manera precisa los intervalos de tiempo en los cuales el MTR ejecuta determinada acción, esto le permite generar un sistema de control de horarios, fundamental para la administración del riego, aplicación de fertilizantes, generación de reportes y demás acciones requeridas para el manejo eficiente de un cultivo.

Para este sistema se seleccionaron componentes electrónicos que en la actualidad son emergentes y de fácil consecución en el mercado, sumado a esto el bajo costo hacen de este módulo una alternativa asequible a los agricultores.

Al incorporar el modem SIM800L y haciendo uso de comandos AT, el módulo puede establecer comunicación GPRS para enviar los datos de los sensores a un servidor web, a través de una URL dispuesta para este fin. Así mismo se puede leer la configuración que haya realizado el usuario desde la plataforma web desarrollada, para cada dispositivo en concreto.

Este trabajo puede extenderse fácilmente a muchos otros campos de acción del sector agroindustrial, ejemplo, como sensor inalámbrico remoto red a través de la integración con redes RF, RFID, control automático industrial, controles de riego, domótica, seguridad, semaforización, entre otros.

\section{AGRADECIMIENTOS}

Los realizadores de este trabajo, agradecemos al servicio nacional de aprendizaje SENA, que financió la propuesta mediante la convocatoria, fomento IDT 2015-2017- Mipymes proceso 1; programa innovación y desarrollo tecnológico productivo en la modalidad de cofinanciación. Se debe mencionar que este proyecto logró el primer lugar en la mencionada convocatoria, con un puntaje de 99/100, compitiendo con más de diez mil propuestas. Igualmente, el agradecimiento a la universidad pedagógica y tecnológica de Colombia "UPTC", y a su programa de ingeniería electrónica, que colaboró con el diseño de un porcentaje importante del hardware desarrollado. Así mismo al centro de desarrollo tecnológico e innovación en TIC, NetworkTIC, S.A.S, quien realizó la ejecución del proyecto; y finalmente a la Universidad "Unitrópico", quien a través del centro de investigación agropecuaria el remanso, permitió realizar la validación del prototipo.

\section{REFERENCIAS}

[1] Z. Münzbergová, V. Hadincová, H. Skálová and V. Vandvik. "Genetic differentiation and plasticity interact along temperature and precipitation gradients to determine plant performance under climate change". Journal of Ecology. Vol. $105 \mathrm{~N}^{\circ}$ 5, pp. 1358-1373. Sep., 2017.

[2] M. Paustian and L. Theuvsen. "Adoption of precision agriculture technologies by German crop farmers". Precision Agriculture. Vol. 18 No 5, pp. 701-716. Oct., 2017.

[3] M.M. Espinoza, R. Isaías, M. Andrade and J.A. Rojas. "Information and communication technologies in agriculture". Revista de Ciencia, Tecnología e Innovación. Vol. 4 $\mathrm{N}^{\circ}$ 1, pp. 105-116. March, 2017.

[4] K.R. Somayeh Tohidyan Far. "Impacts of the precision agricultural technologies in Iran: An analysis experts' perception \& their determinants". Information Processing in Agriculture. Vol. 3, pp. 73-84. 2018. 
[5] M.N.T. Ebrahim Babaeian. "A New Optical Remote Sensing Technique for HighResolution Mapping of Soil Moisture". Frontiers in Big Data. 2019.

[6] Corpoica. "Plan estratégico de ciencia, tecnología e innovación del sector agropecuario colombiano - PECTIA”. Bogota, p. 33. 2017.

[7] D.L. Rincón Ballesteros, J.E. Fonseca Ramírez y J.A. Orjuela-Castro. "Hacia un Marco Conceptual Común sobre Trazabilidad en la Cadena de Suministro de Alimentos". Ingeniería. Vol. $22 \mathrm{~N}^{\circ}$ 2, p. 161. May 2017.

[8] F.L. Jorge, E. Chaparro y Nelson Barrera Lombana. "Módulo de agrometría basado en plataforma Android y Bluetooth RN42, 'AgroAPP'”. Revista Gerencia en Tecnología, Informática. Vol. $15 \mathrm{~N}^{\circ}$ 41, pp. 17-31. 2016.

[9] J.S. Rueda and J.M. Talavera Portocarrero. "Similitudes y diferencias entre Redes de Sensores Inalámbricas e Internet de las Cosas: Hacia una postura clarificadora". Revista Colombiana de Computación. Vol. $18 \mathrm{~N}^{\circ} 2$, pp. 58-74. Dec., 2017.

[10] M.C. Vuran, A. Salam, R. Wong and S. Irmak. "Internet of underground things in precision agriculture: Architecture and technology aspects". Ad Hoc Networks. Vol. 81, pp. 160-173. Dec., 2018.

[11] M. Mamani, M. Villalobos y R. Herrera. "Sistema web de bajo costo para monitorear y controlar un invernadero agrícola". Ingeniare. Revista chilena de ingeniería. Vol. $25 \mathrm{~N}^{\circ} 4$, pp. 599-618. 2017.

[12] J.D.S. Álvaro Hernán Alarcón, Geyni Arias y Cristian Javier Díaz. "Sistema de control automático de variables climáticas para optimizar el rendimiento de cultivos bajo cubiertas". Ingeniería Solidaria. Vol. $14 \mathrm{~N}^{\circ} 24$, pp. 12-25. 2018.

[13] J. Parada y J. Carrillo. "Automatización de sistemas de riego: estrategias de control a través de dispositivos móviles". Renovat: Revista de Estudios Interdisciplinarios en Ciencias Sociales, Tecnología e Innovación. No 1, pp. 138-160. Dec., 2016.

[14] J. Puentes Rivero y B.A. Baquero Cardozo. "Dispositivo para monitorear en tiempo real vía web variables como temperatura humedad y concentración de $\mathrm{CO}_{2}$ que puedan afectar la calidad de la mercancía durante su transporte". Revista chilena de ingeniería. Vol. 10 N$^{\circ}$ 45, pp. 9-17. 2017.
[15] A. Fernández. "Remote supervision and control based on wireless technology to operation of central pivot irrigation machine". Sistemas y Telemática. Vol. $16 \mathrm{~N}^{\circ} 44$, pp. 63-74. Feb., 2018.

[16] E.A. Maya Olalla y G.X. Chafla Altamirano. "Red inalámbrica de sensores a través de 6lowpan para una agricultura de precisión". SATHIRI: Sembrador. $N^{\circ}$ 6, pp. 22-32. July, 2018.

[17] F. Mendoza Mondragón. "Sistema de monitoreo y control de invernaderos a través de una red inalámbrica mediante un servidor web embebido en microcontroladores de alto rendimiento". 2010.

[18] X. Zhu, D. Li, D. He, J. Wang, D. Ma and F. Li. "A remote wireless system for water quality online monitoring in intensive fish culture". Computers and Electronics in Agriculture. Vol. 71, pp. 3-9. 2010.

[19] K. Pigeon. "User Manual gsm 3g m2m RTU Automation solution, King Pigeo". China provincia de Guangdong: Automation solution. 2018.

[20] C.J. Pérez Bladimir, Koo José y F. Garcia. "Automatización, monitoreo y control remoto de un sistema de riego agrícola con código abierto". Latin American and Caribbean Conference for Engineering and Technology. Vol. 12, No April, pp. 1-9. 2014.

[21] N.B. Jorge Chaparro. "Módulo de agrometría basado en plataforma Android y Bluetooth rn42, 'AgroAPP'". Revista Gerencia en Tecnología. Informática. Vol. $15 \mathrm{~N}^{\mathrm{o}} 41$, pp. 17-31. Nov. 2016.

[22] T. Semeraro, G. Mastroleo, A. Pomes, A. Luvisi, E. Gissi and R. Aretano. "Modelling fuzzy combination of remote sensing vegetation index for durum wheat crop analysis". Computers and Electronics in Agriculture. Vol. 156 N $^{\circ}$ December 2018, pp. 684-692. 2019.

[23] A. Jiménez-López, F. Jiménez-López y D. García-Ramírez. "Software para el estudio de coberturas vegetales con conceptos de agricultura de precisión". Ingenio Magno. Vol. $6 \mathrm{~N}^{\circ} 1$, pp. 128-139. 2016.

[24] J.P. Monzon, P.A. Calviño, V.O. Sadras, J.B. Zubiaurre and F.H. Andrade. "Precision agriculture based on crop physiological principles improves whole-farm yield and profit: A case study". European Journal of Agronomy. Vol. 99, pp. 62-71. Sep., 2018. 
[25] J.A.T.C. José Ignacio Rodríguez Molano. "Metamodelo de Integración de Internet de las Cosas y la Industria 4.0.: La Universidad Pedagógica y Tecnológica de Colombia”. Proceedings of the 12th Iberian Conference on Information Systems and Technologies. 2017.

[26] J.G.F. Mendoza, J.A.A. España, J.B.M. Castro, J.G.D. Núñez y E.N. Vaca. "Propuesta metodológica para validar la funcionalidad de software en sistemas embebidos". Pistas Educativas. Vol. 38 N $^{\circ}$ 122. Dec., 2016.

[27] L. González y G.U. Giraldo. "Modelo de requisitos para sistemas embebidos". Revista Ingenierías Universidad de Medellín. Vol. 7 $\mathrm{N}^{\circ} 13$, pp. 111-127. 2008.

[28] M.A. Tosini et al. "Metodologías de Diseño para Sistemas Embebidos". XV workshop de investigadores en ciencias de la computación, pp. 713-717. 2013.

[29] J.J. Morales Arias and C.J. Pardo Calvache. "Systematic review about the integration of model-driven software development and agile methodologies". Informador Técnico. UPTC. Vol. $80 \mathrm{~N}^{\circ}$ 1, p. 87. Jan., 2016.

[30] J.R. Molina Ríos, M.P. Zea Ordóñez, M.J. Contento Segarra y F.G. García Zerda. "Estado del arte: metodologías de desarrollo en aplicaciones web". Revista Ciencia e Ingeniería. Vol. $6 \mathrm{~N}^{\circ}$ 3, p. 54. Sep., 2017.

[31] M.R. Mollahoseini Ardakani, S.M. Hashemi and M. Razzazi. "Adapting the scrum methodology for establishing the dynamic inter-organizational collaboration". Journal of Organizational Change Management. Vol. $31 \mathrm{~N}^{\circ}$ 4, pp. 852-866. July, 2018.

[32] Incorporates IEEE. "1012-2016 Standard IEEE for System, Software, and Hardware Verification and Validation". IEEE. 2017.

[33] Microchip. "pic18F4620. Data Sheet". 2018. [Online]. URL: http://pdf1.alldatasheet.com/ datasheet-pdf/view/115982/MICROCHIP/ PIC18F4620.html. Accessed: 14-Sep-2018.

[34] Dallas Semiconductor. "DS18B20 Datasheet (PDF) - Dallas Semiconductor". 2018. URL: http://www.alldatasheet.com/datasheet$\mathrm{pdf} / \mathrm{pdf} / 58557 / \mathrm{DALLAS} / \mathrm{DS} 18 \mathrm{~B} 20 . \mathrm{html}$. Accessed: 18-Jan-2019.

[35] Alldatasheetcom. "Relative humidity sensor HS1100/HS 1101". Hd humiriel, p. 4. 2017.

[36] Dallas Semiconductor. "DS 12887 Datasheet(PDF) - Dallas Semiconductor". Dallas Semiconductor. 2018. URL: http:// www.alldatasheet.com/datasheet-pdf/ pdf/58474/DALLAS/DS12887.html. Accessed: 18-Jan-2019. 